\title{
Bullying Perpetration and Victimization among Adolescents with Overweight and Obesity in a Nationally Representative Sample
}

\author{
Kristie Rupp, $\mathrm{PhD}^{\prime}$ and Stephanie M. McCoy, PhD, MPH ${ }^{2}$
}

\section{Abstract}

Background: Adolescents with obesity are more likely to experience bullying in comparison to their healthy weight peers. However, it is unclear whether adolescents with obesity are more likely to perpetuate bullying or be both, a bully perpetrator and a bully victim. The purpose of this analysis was to examine differences in bully perpetration, victimization, and both (perpetration and victimization) by BMI classification in a nationally representative sample of adolescents.

Methods: Analyses included 31,770 adolescents, ages 10-17, from the combined 2016-2017 National Survey of Children's Health. Adolescents were grouped by BMI classification; outcome variables included bullying, difficulty making new friends, excessive arguing, depression, and behavioral conduct problems. Logistic regression models, adjusted for age, sex, race, household income, highest level of education in the household, and attention-deficit/hyperactivity disorder assessed the odds of each outcome comparing healthy weight to adolescents with overweight and obesity.

Results: Adolescents with overweight and obesity had greater odds of experiencing bullying behaviors: bully victim [odds ratio $(\mathrm{OR})=1.34$ and 2.03] and both bully perpetrator and victim $(\mathrm{OR}=1.37$ and 2.01$)(p$ 's $<0.05)$, respectively, in comparison to healthy weight peers. Adolescents with overweight or obesity involved in bullying behaviors had significantly higher odds of behavioral conduct problems, depression, arguing excessively, and having difficulty making friends compared to adolescents with overweight or obesity who were neither a bully perpetrator nor victim $(p$ 's $<0.05)$.

Conclusions: To promote overall health and well-being among adolescents with overweight and obesity, effort should be made to mitigate engagement in and/or victimization from bullying and associated behavioral or depressive symptoms.

Keywords: adolescence; bullying; obesity

\section{Introduction}

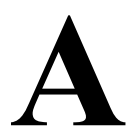

dolescents with overweight and obesity are more likely to be victims of bullying in comparison to their healthy weight peers. ${ }^{1}$ While adolescents with overweight and obesity experience higher rates of all forms of bullying, including weight-based, race-based, socioeconomic (SES)-based, and sexual harassment; ${ }^{2}$ weightbased victimization by peers is the most commonly reported form of bullying. ${ }^{1,3}$ Furthermore, adolescents (of all weight classes) perceive weight-status to be the primary reason their peers are bullied in comparison to other reasons for bullying. ${ }^{4}$ The high rates of peer victimization among adolescents with overweight and obesity may negatively affect social and emotional health and well-being in addition to the established physical health effects of overweight and obesity.
One study by Puhl and Luedicke, found that bully victimization, particularly weight-based victimization, is associated with a $40 \%-50 \%$ greater likelihood of feeling sad, depressed, and worse about themselves. ${ }^{5}$ Moreover, weightrelated bullying by peers may lead to barriers in developing peer relationships and may further social isolation among adolescents with overweight and obesity, particularly among female adolescents. ${ }^{1,6}$ Thus, adolescents with overweight and obesity are particularly susceptible to bullying by peers, particularly weight-based victimization, which may negatively affect their emotional health and ability to develop peer relationships and engage socially during a key period in development.

While there is considerable evidence that adolescents with overweight and obesity are more likely to be victims of bullying in comparison to their healthy weight peers, the

'Department of Kinesiology, Brooklyn College of the City University of New York, Brooklyn, NY.

${ }^{2}$ School of Kinesiology and Nutrition, University of Southern Mississippi, Hattiesburg, MS. 
likelihood of bully perpetration in adolescents with overweight and obesity is less clear. ${ }^{7,8}$ One study found that 15 to 16-year-old adolescents were more likely than their healthy weight peers to perpetuate bullying, but did not see this relationship among 11- to 14-year-old adolescents. ${ }^{7}$ Another study by Odar Stough et al., found that adolescents with overweight and obesity were significantly more likely to perpetuate bullying than their healthy weight peers in unadjusted analyses, but this relationship did not remain significant when controlling for other child, family, and neighborhood factors. ${ }^{8}$ Adolescents who engage in bullying behavior are at a greater risk of experiencing externalizing and internalizing problems, including depressive symptoms, and are more likely to experience substance abuse problems in the future,,${ }^{9,10}$ thus highlighting the negative psychological consequences experienced by both bully perpetrators and bully victims.

While most studies have examined the effects of bully victims and bully perpetrators separately, few studies have examined the likelihood of being both a bully victim and bully perpetrator among adolescents with overweight and obesity. One study by Jansen et al. of teacher-reported bullying behaviors found that children (mean age 6.2 years) with obesity (BMI $\geq 95$ th percentile) were significantly more likely to be bully victims and perpetrators in comparison to their healthy weight peers. ${ }^{11}$ Another study by Bacchini et al., in a population of outpatient children (ages 6 to 14 years), showed boys with severe obesity were at a higher risk for being both a bully victim and bully perpetrator. ${ }^{12}$ However, these studies both examined children and young adolescents. Given bullying behavior tends to decline as children age, ${ }^{13}$ it is unknown whether adolescents with overweight and obesity are at a greater risk of being both a bully victim and bully perpetrator.

Engagement in bullying as a victim, perpetrator, or both, is associated with worse psychological and physical health regardless of weight status. ${ }^{13,14}$ There is a dearth of literature on bullying behavior among adolescents with overweight and obesity. Previous studies that have examined all three bully classifications (likelihood of being a bully victim, bully perpetrator, or both a bully victim and bully perpetrator) have done so in younger children with overweight and obesity, ${ }^{11,12}$ which warrants further examination among adolescents. The purpose of this study was to examine the differences in perpetration, victimization, and both (perpetration and victimization), by weight classification in a nationally representative sample of adolescents. Given the adverse psychological outcomes associated with bullying behaviors, ${ }^{13-15}$ we additionally wanted to determine the odds of experiencing behavioral conduct problems, depression, excessive arguing, and difficulty making or keeping friends among adolescents with overweight or obesity, stratified by bullying classification (perpetrator, victim, or both). We hypothesized that adolescents with overweight and obesity would have greater odds of bully perpetration, victimization, and both perpetration and victimization. We hypothesized that among adolescents with overweight and obesity, bully perpetrators, victims, and perpetrator/victims would have greater odds of experiencing behavioral conduct problems, depression, excessive arguing, and greater difficulty making and keeping friends compared to adolescents with overweight and obesity who were classified neither as perpetrator nor as victim.

\section{Methods}

\section{Data Source}

We used de-identified data from the combined 20162017 National Survey of Children's Health (NSCH), ${ }^{16}$ a cross-sectional national survey, for our analyses. The $\mathrm{NSCH}$ includes data on physical and mental health, access to and quality of health care, as well as information on the adolescent's family, neighborhood, school, and social context. The NSCH is sponsored by the Maternal and Child Health Bureau within the Health Resources and Services Administration, United States Department of Health and Human Services and in 2016 was administered via web and mail (paper) based instruments by the US Census Bureau. Households were randomly contacted to identify households with children younger than the age of 18 years. If a household had more than one child, one was randomly selected, and parents/guardians completed the questionnaire. The overall response rate was $40.7 \%$. Further study design details are available elsewhere. ${ }^{16,17}$

\section{Participants}

The sample was limited to adolescents between the ages of 10 and 17 years $(n=37,409)$, to be consistent with the World Health Organization definition of adolescence, $10-19$ years. ${ }^{18}$ In addition, adolescents were excluded from the analysis if they had missing data for any primary variables, including BMI classification $(n=2165)$; bully perpetration $(n=573)$; bully victimization $(n=188)$; difficulty making friends $(n=235)$, excessive arguing $(n=64)$; depression $(n=166)$; and behavioral conduct problems $(n=143)$. Those classified as underweight (BMI $\leq 5$ th percentile) were excluded as the purpose of the study was to determine associations between adolescents with overweight or obesity compared to healthy weight peers; yielding a final sample size of $(n=31,770)$.

\section{Measures}

BMI classification. BMI classification served as the independent variable in our primary analyses, and was calculated from parent-reported height and weight and categorized as recommended by the CDC: healthy weight (BMI $>5$ th percentile and $<85$ th percentile); overweight (BMI $\geq 85$ th percentile and $<95$ th percentile); and obese (BMI $\geq 95$ th percentile). ${ }^{19}$ For the purpose of this study, only BMI classification was used, as height and weight were not available in the dataset.

Bullying. Parents were asked "how well do each of the following phrases describe this child: (1) this child is bullied, picked on, or excluded by other children; and (2) 
this child bullies others, picks on them, or excludes them." Parent responses were recorded on a three-point scale ("definitely true, somewhat true, or not true"). Responses were further dichotomized to indicate exhibition of the behavior. "Definitely true" and "somewhat true" were categorized as "yes," a "not true" was recorded as a "no," similar to previous literature utilizing the NSCH data set. ${ }^{20}$

A four-level composite bullying variable was generated from the survey items examining bully perpetration and victimization. Responses from the bullying items listed in the previous paragraph were used to generate a new variable which classified each adolescent into one of four categories: "neither perpetrator nor victim $(n=24,095)$ " (those participants with a "not true" response to both the bullying others and bully victim items), "perpetrator $(n=461)$ " (those participants with a "definitely true" or "somewhat true" response to the bullying others item and a "not true" response to the bully victim item), "victim $(n=6085)$ ", (those participants with a "not true" response to the bullying others item and a "definitely true" or "somewhat true" response to the bully victim item), or "both perpetrator and victim $(n=1129)$ )" (those participants with a "definitely true" or "somewhat true" response to both the bullying others and bully victim items).

Excessive arguing. Parents were asked on a three-point scale ("definitely true, somewhat true, or not true") if their child argues too much. Responses were further dichotomized to indicate exhibition of a positive indicator. "Definitely true" and "somewhat true" were categorized as "yes," a "not true" was recorded as a "no" to remain consistent with dichotomization across variables utilizing the three point Likert scale.

Difficulty in making new friends. Parents were asked "compared to other children of his or her age, how much difficulty does this child have making or keeping friends?" Parent responses were recorded on a three-point scale ("no difficulty, a little difficulty, or a lot of difficulty"). Responses were further dichotomized to indicate exhibition of a positive indicator. "A little difficulty" and "a lot of difficulty" were categorized as "yes," and "no difficulty" was recorded as a "no."

Behavioral conduct problems. Parents were asked "has a doctor, other health care provider, or educator ever told you that your child has behavioral or conduct problems?" Parent responses were recorded as either a "yes" or "no." If a yes response was recorded for behavioral conduct problems, parents were further asked "does this child currently have the condition?" Responses were classified as "yes", if parents responded that their child currently had the condition.

Depression. Parents were asked "has a doctor, other health care provider, or educator ever told you that your child has depression?" Parent responses were recorded as either a "yes" or "no." If a yes response was recorded for depression, parents were further asked "does this child currently have the condition?" Responses were classified as "yes", if parents responded that their child currently had the condition.

\section{Statistical Analyses}

Analyses were completed using Stata 15.1 (Stata Corporation, College Station, TX). Chi-square tests were used to compare the prevalence of sociodemographic characteristics by BMI classification. An adjusted multinomial logistic regression was used to determine the associations between BMI classification and bullying classification: perpetration, victimization, and both (perpetration and victimization). Secondary analyses using adjusted logistic regression models were conducted to determine associations between bullying classification and secondary outcomes of interest: behavioral conduct problems, depression, excessive arguing, and difficulty making friends among adolescents with overweight and obesity. Each model was adjusted for the covariates: age, sex, race, household income (multiply imputed data for missing data), highest level of education in the household, and the presence of ADD/attention-deficit/ hyperactivity disorder (ADHD). Sociodemographic covariates were chosen based on the potential for confounding and previous literature. ${ }^{10,21,22}$ ADHD was chosen as a covariate as it has been shown that there is an association between obesity and ADHD as individuals age into adolescence and adulthood. ${ }^{23}$ In addition, individuals with ADHD are significantly more likely to report being victimized by peers. ${ }^{24}$

\section{Results}

Analyses included 31,770 adolescents; $~ 71 \%$ of adolescents were healthy weight, $15 \%$ were overweight, and $14 \%$ were obese. Demographic characteristics by BMI classification are presented in Table 1. Compared to healthy weight adolescents, adolescents with overweight or obesity were slightly younger [mean \pm standard deviation; $13.6 \pm 2.3$ years and $13.7 \pm 2.3$ years, respectively, vs. $14.0 \pm 2.2$ years; $p<0.001]$, more likely to be male $(51.6 \%$ and $60.4 \%$, respectively, vs. $48.2 \% ; p<0.001)$, and less likely to be nonHispanic white $(76.4 \%$ and $74.2 \%$, respectively, vs. $79.8 \%$; $p<0.001)$. In addition, compared to healthy weight adolescents, adolescents with overweight and obesity were more likely to live in a household at $<133 \%$ of the federal poverty level $(15.5 \%$ and $21.6 \%$, respectively, vs. $11.6 \%$; $p<0.001)$ and less likely to have a guardian with more than 12 years of schooling $(82.2 \%$ and $74.9 \%$, respectively, vs. $87.2 \% ; p<0.001)$. Furthermore, compared to healthy weight adolescents, a higher percentage of adolescents with overweight or obesity were involved in bullying behaviors as both a bully perpetrator and victim $(4.2 \%$ and $5.7 \%$, respectively, vs. $3.0 \% ; p<0.001)$. A lower percentage of adolescents with overweight and obesity were neither a bully perpetrator nor a victim $(72.7 \%$ and $63.7 \%$, respectively, vs. $78.8 \% ; p<0.001)$. 


\begin{tabular}{|c|c|c|c|c|}
\hline Characteristic & Healthy weight $(n=22,633)$ & Overweight $(n=4816)$ & Obese $(n=432 I)$ & $p$ \\
\hline Percentage of sample & 71.2 & 15.2 & 13.7 & \\
\hline Age (years) M [SD] & $14.0[2.2]$ & I3.6 [2.3] & $13.7[2.3]$ & $<0.001$ \\
\hline Sex (male) [\%] & 48.2 & 51.6 & 60.4 & $<0.001$ \\
\hline Race, White non-Hispanic (\%) & 79.8 & 76.4 & 74.2 & $<0.001$ \\
\hline Household income ${ }^{\mathrm{a}}(\%)$ & & & & $<0.001$ \\
\hline Poor & 11.6 & 15.5 & 21.6 & \\
\hline Near poor & 7.0 & 8.5 & 10.4 & \\
\hline Not poor & 81.4 & 75.9 & 68.0 & \\
\hline Highest education ${ }^{\mathrm{b}}$ (\%) & & & & $<0.001$ \\
\hline$<12$ years & 1.6 & 2.9 & 4.1 & \\
\hline 12 years & 11.2 & 14.9 & 21.0 & \\
\hline$>12$ years & 87.2 & 82.2 & 74.9 & \\
\hline Bullying behaviors & & & & $<0.001$ \\
\hline Neither bully perpetrator nor victim & 78.8 & 72.7 & 63.7 & \\
\hline Perpetrator & 1.5 & 1.5 & 1.6 & \\
\hline Victim & 16.7 & 21.6 & 29.0 & \\
\hline Both bully perpetrator and victim & 3.0 & 4.2 & 5.7 & \\
\hline
\end{tabular}

Statistics represents between group differences. $p<0.05$ in boldface.

aIncome is grouped based on DHHS Federal Poverty Guidelines for households: "poor," <133\% poverty; "near poor," $\geq 133 \%$ poverty but $<185 \%$ poverty; "not poor," $\geq 185 \%$ poverty.

bHighest level of education in family.

$\mathrm{SD}$, standard deviation.

For adolescents with overweight and obesity, the adjusted odds of experiencing bullying behaviors were significantly higher than their healthy weight peers (Table 2). Adolescents with overweight had $34 \%$ greater odds of being a bully victim [odds ratio $(\mathrm{OR})=1.34,95 \%$ confidence interval (CI): (1.24-1.45)], and 37\% greater odds of being both a perpetrator and a victim $[\mathrm{OR}=1.37,95 \% \mathrm{CI}$ : (1.16-1.61)]. In addition, in adolescents with obesity, the odds of being a bully victim were 2.03 times greater $[\mathrm{OR}=2.03,95 \% \mathrm{CI}:(1.88-2.20)]$ and 2.01 times greater to be both a perpetrator and a victim $[\mathrm{OR}=2.01,95 \% \mathrm{CI}$ : (1.72-2.35)] compared with their healthy weight peers.

\section{Table 2. Adjusted ${ }^{a}$ Odds Ratios of Bullying Behaviors by BMI Classification}

\begin{tabular}{|c|c|c|c|c|c|}
\hline \multirow[b]{2}{*}{ Variable } & \multirow{2}{*}{$\begin{array}{l}\text { Healthy weight } \\
\quad(n=22,633)\end{array}$} & \multicolumn{2}{|c|}{ Overweight $(n=4816)$} & \multicolumn{2}{|c|}{ Obese $(n=4321)$} \\
\hline & & OR & $95 \% \mathrm{Cl}$ & OR & $95 \% \mathrm{Cl}$ \\
\hline \multicolumn{6}{|l|}{ Bully behaviors } \\
\hline Neither bully perpetrator nor victim & Reference & - & & - & \\
\hline Perpetrator & Reference & 1.10 & {$[0.85-1.43]$} & 1.30 & {$[0.99-1.70]$} \\
\hline Victim & Reference & 1.34 & {$[1.24-1.45]$} & 2.03 & {$[1.88-2.20]$} \\
\hline Both bully perpetrator and victim & Reference & 1.37 & {$[1.16-1.61]$} & 2.01 & [1.72-2.35] \\
\hline
\end{tabular}

$p<0.05$ in boldface.

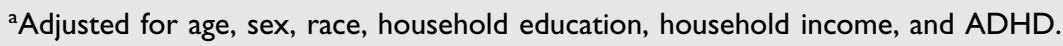

$A D H D$, attention-deficit/hyperactivity disorder; OR, odds ratio; $\mathrm{Cl}$, confidence interval. 


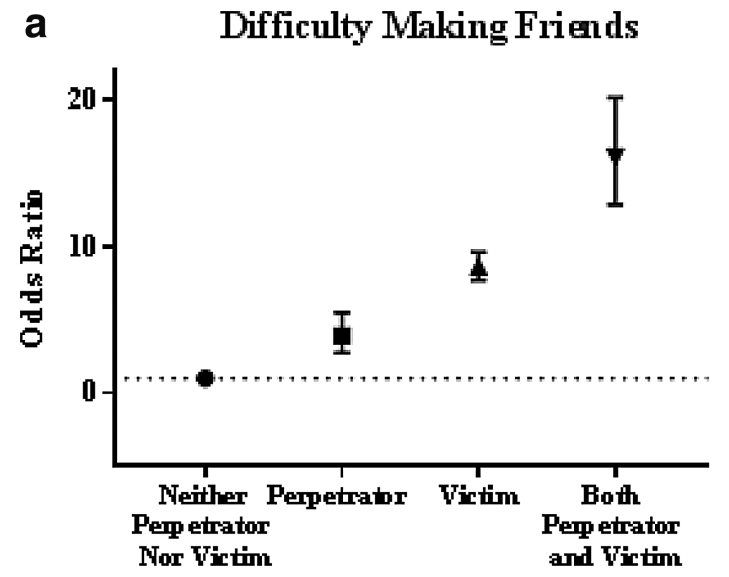

Bully Classification

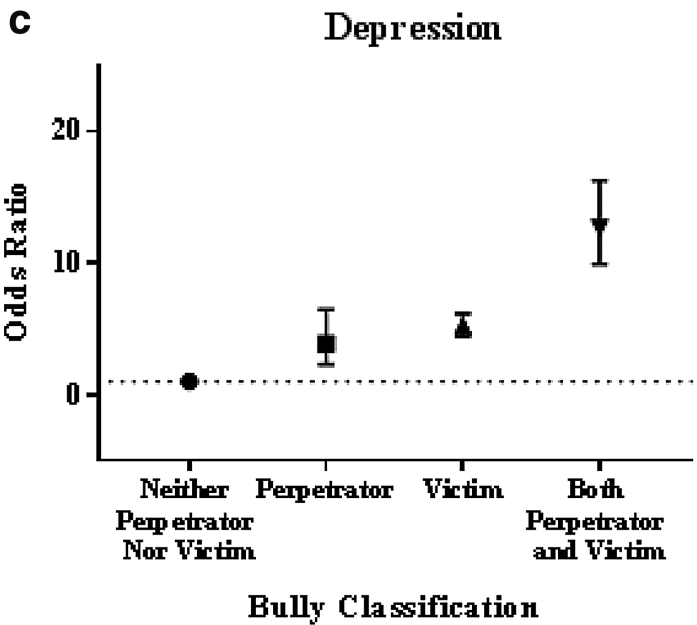

b

Behavior al Problems

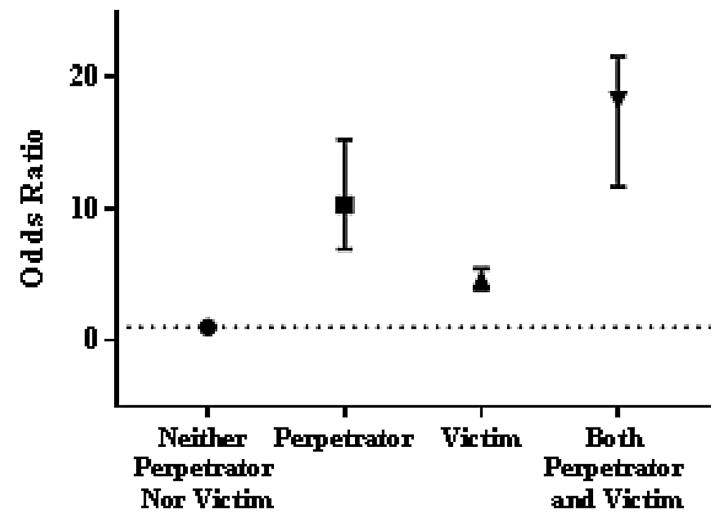

Bully C lassification

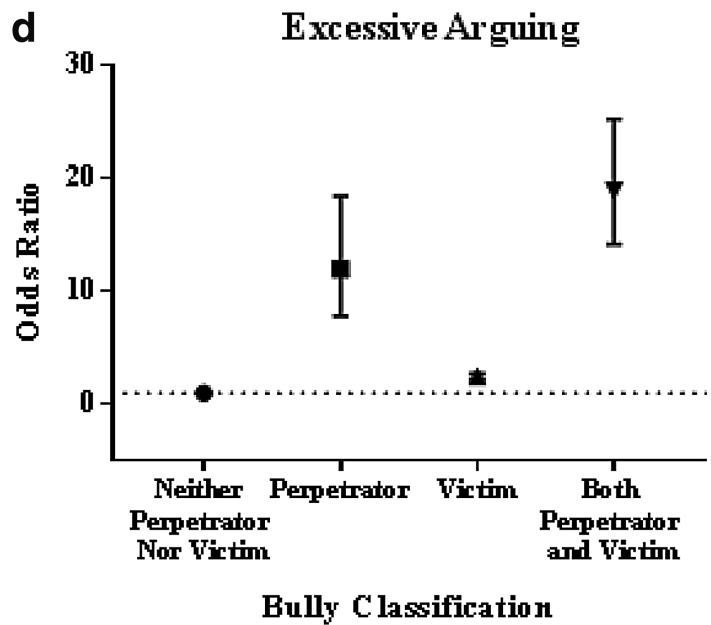

Figure 1. Adjusted odds ratios of (a) Difficulty making friends, (b) Behavioral conduct problems, (c) Depression, and (d) Excessive arguing by bully classification among adolescents with overweight and obesity. Error bars represent $95 \%$ confidence intervals.

Odds of being a bully perpetrator were not statistically significant in adolescents with overweight $[\mathrm{OR}=1.10,95 \%$ CI: (0.85-1.43)] nor in adolescents with obesity [OR $=1.30$, 95\% CI: (0.99-1.70)] compared with healthy weight peers.

As shown in Figure 1 (Supplementaty Table S1), adolescents with overweight or obesity who were a bully perpetrator, victim, and both perpetrator and victim had significantly higher odds of experiencing behavioral conduct problems, depression, arguing excessively, and having difficulty making friends compared with adolescents with overweight or obesity who are neither a bully perpetrator nor a victim. Those classified as both, a bully perpetrator and victim, had demonstratively higher odds of experiencing these outcomes.

\section{Discussion}

This study sought to determine the odds of (1) engaging in three distinct categories of bullying behavior (bully victim, bully perpetrator, and both bully victim and perpetrator) in adolescents with overweight and obesity in comparison to their healthy weight peers and (2) experi- encing social, emotional, and behavioral problems in adolescents with overweight and obesity, who are engaged in bullying behaviors. Partially confirming our initial hypothesis that adolescents with overweight and obesity have significantly higher odds of being a bully victim and being both a bully victim and a bully perpetrator; however, adolescents with overweight and obesity did not have significantly higher odds of being a bully perpetrator. Our finding that adolescents with overweight and obesity have significantly greater odds in comparison to their healthy weight peers of being victims of bullying confirms findings of previous literature indicating a relationship between elevated weight status and peer victimization. ${ }^{25-28}$ In a previous analysis of the 2011-2012 NSCH data set, Odar Stough et al. examined bully perpetration in adolescents with overweight and obesity, but they did not examine bully victimization and both, bully perpetration and victimization as the question about bully victimization was not available in the 2011-2012 NSCH data set. ${ }^{8}$ Similar to Odar Stough et al., we also found that adolescents with overweight and obesity do not have significantly greater 
odds of perpetrating bullying than their healthy weight peers after adjustment for other relevant demographic factors. ${ }^{8}$ Our results build upon the findings of Odar Stough et al., ${ }^{8}$ by demonstrating that adolescents with overweight and obesity have significantly higher odds of being a bully victim and both, a bully victim and a perpetrator in comparison to their healthy weight peers.

While we did not detect significantly higher odds of bully perpetration only among adolescents (ages 10-17 years) with overweight or obesity, Janssen et al., in a crosssectional analysis of the Health Behaviour in School-Aged Children Survey, found that 15- to 16-year-old adolescents with obesity were significantly more likely to perpetuate bullying compared with their healthy weight peers. $^{7}$ However, this relationship was not significant among 11to 14 -year-olds, ${ }^{7}$ thus, it is possible that since our sample included adolescents aged 10-17 years and examining potential differences in behavior across different stages of adolescence was beyond the scope of the current analysis, we were unable to detect a higher odds of bully perpetration that may be present among older adolescents. It also must be considered that our study examined bullying behaviors (perpetration and victimization) separately and together, which may help explain why previous studies have had mixed findings in the odds of bully perpetration among adolescents with overweight and obesity. ${ }^{7,8,10}$ Furthermore, it is possible that the relatively low sample size of adolescents with overweight or obesity who were bully perpetrators only may have limited our ability to detect a significant difference.

Our study demonstrated, in a nationally representative sample, that adolescents with overweight and obesity have significantly greater odds of being both a bully victim and a bully perpetrator. Bacchini et al. found in a sample of treatment seeking Italian youth, aged 6-14 years, significantly greater odds of being both a bully and bully-victim in those with severe obesity (BMI-standard deviation score $>2.5$ ) only. ${ }^{12}$ Similarly, Jansen et al. found in a national sample of children from the Netherlands, mean age 6.2 years, an increased likelihood of being both a bully victim and a perpetrator in children with obesity, but not with overweight. ${ }^{11}$ Our study expands upon these findings, by demonstrating that there are increased odds of being both a bully victim and a bully perpetrator among a nonobesity treatment seeking population, in an older age range of adolescents, and among adolescents with overweight as well. This finding is of particular interest due to the higher rates of emotional and behavioral issues associated with concurrent bully victim and perpetration as demonstrated by our secondary analyses.

Our secondary hypotheses were also confirmed; there were significantly greater odds of experiencing behavioral conduct problems, depression, excessive arguing, and difficulty making friends in adolescents with overweight and obesity who are engaged in bullying behaviors as a perpetrator, victim, or both, in comparison with those who are not involved in bullying behaviors. Our findings build upon previous studies, which have found lower self-esteem; higher depressive symptoms ${ }^{29}$; greater conduct, school, and social problems ${ }^{15}$; and more frequent physical and emotional symptoms ${ }^{13}$ among adolescents who are involved in bullying behaviors in any capacity, by specifically demonstrating this relationship in adolescents with overweight and obesity. Furthermore, we saw increased odds of experiencing these behavioral, emotional, and social problems in adolescents with obesity who were both bully victims and bully perpetrators. Our results are in contrast to the findings of Juvonen et al., who found that bullies were psychologically stronger (e.g., lower levels of depression, loneliness, and social anxiety) than students not involved in bullying. ${ }^{15}$ However, confirming our findings, Juvonen et al. also found that students who were engaged in bullying as both (perpetrator and victim) exhibited the highest levels of misconduct, as well as school and peer relationship problems among the three bullying groups (perpetrator, victim, and both). ${ }^{15}$ The difference in our results might be explained by the difference in age ranges or the population sampled. Juvonen et al. sampled younger adolescents from lower socioeconomic status urban communities,${ }^{15}$ whereas our sample was nationally representative and our observations were made specifically in a sample of adolescents with overweight or obesity.

Our study had several strengths, including (1) examination of three distinct classifications of bullying behavior in adolescents with overweight and obesity; (2) utilization of a large nationally representative sample; and (3) examination of bullying-associated social, emotional, and behavioral problems specifically within a population of adolescents with overweight and obesity. Nevertheless, there are some limitations of this study that should be considered when interpreting the findings of this study. The data collected in the NSCH are all parent-reported, which may lead to misclassification of BMI, bullying behaviors, or behavioral, emotional, and social problems. However, it is recognized that parents have the greatest knowledge of their child's health, and parental report is a valid proxy measurement for child health; inclusive of height, weight, and health-related quality of life. ${ }^{30,31}$ With respect to parent-reported weight, parents are significantly more likely to underestimate weight among adolescents leading to an underestimation of overweight and obesity. ${ }^{30}$ Furthermore, parents are more likely to underestimate weight as BMI $z$-score increases, ${ }^{32}$ thus it is highly likely that reported BMI classifications are underestimated in this analysis potentially attenuating the results of this study. Moreover, since parents only spend part of the day with their day with their child, it is possible that reported bully victimization or perpetration is likely underestimated. These potential parent-reported underestimations only bring further validity to our findings. It must also be considered that the data collected from the NSCH do not provide specifics on the type of bullying behaviors experienced by the adolescent (e.g., overt, relational, verbal, and cyber), which may limit generalizability to specific types of bullying in 
this population. Future studies are needed to elucidate the types of bullying behaviors adolescents with overweight and obesity are most likely to be engaged in.

Since our study was cross-sectional, we are not able to determine the causality of our findings. However, previous longitudinal studies have found that victimization from bullying is predictive of worse future psychological health. ${ }^{14,33}$ Furthermore, Copeland et al. found that engagement in bullying behaviors as both a perpetrator and a victim in childhood and adolescence was predictive of more severe psychiatric outcomes in young adulthood, ${ }^{33}$ similar to what we observed in our cross-sectional analysis. Although we cannot rule out that reverse causality may explain some of our findings, longitudinal studies are needed to establish long-term social, behavioral, and emotional consequences of experiencing bullying behaviors in adolescents with overweight and obesity.

\section{Conclusion}

Our study demonstrates increased odds of being a bullyvictim and both, a bully victim and bully perpetrator, among a nationally representative sample of adolescents with overweight and obesity, expanding upon previous studies on bullying behaviors in adolescents. ${ }^{8,11,12}$ Furthermore, our findings further strengthen the relationship between behavioral, emotional, and social problems and bullying behaviors, ${ }^{13,15,29}$ by establishing this relationship within a specific population of adolescents with overweight and obesity. Moreover, we found increased odds of these associated behavioral, emotional, and social problems in those who are both a bully victim and a bully perpetrator in comparison to adolescents with overweight or obesity, who are not involved in bullying behaviors. Thus, our study highlights the need for awareness and detection of bullying behaviors, specifically as both a bully victim and a perpetrator, to effectively address underlying behavioral, social, and emotional problems to promote overall health and well-being in adolescents with overweight and obesity.

\section{Acknowledgments}

All work for this study, including requesting data, statistical analysis, and article preparation was conducted equally by K.R and S.M.M. This research did not receive any specific grant from funding agencies in the public, commercial, or not-for-profit sectors. The authors declare no conflicts of interest.

\section{Author Disclosure Statement}

Author's K.R. and S.M.M. declare that no competing financial interests exist.

\section{Supplementary Material}

\footnotetext{
Supplementary Table S1
}

\section{References}

1. Hayden-Wade HA, Stein RI, Ghaderi A, et al. Prevalence, characteristics, and correlates of teasing experiences among overweight children vs. non-overweight peers. Obesity 2005;13:1381-1392.

2. Bucchianeri MM, Eisenberg ME, Neumark-Sztainer D. Weightism, racism, classism, and sexism: Shared forms of harassment in adolescents. $J$ Adolesc Health 2013;53:47-53.

3. Fonseca H, Matos MG, Guerra A, et al. Are overweight and obese adolescents different from their peers? Pediatr Obes 2009;4:166-174.

4. Puhl RM, Luedicke J, Heuer C. Weight-based victimization toward overweight adolescents: Observations and reactions of peers. $J$ Sch Health 2011;81:696-703.

5. Puhl RM, Luedicke J. Weight-based victimization among adolescents in the school setting: Emotional reactions and coping behaviors. J Youth Adolesc 2012;41:27-40.

6. Griffiths LJ, Wolke D, Page AS, et al. Obesity and bullying: Different effects for boys and girls. Arch Dis Child 2006;91:121-125.

7. Janssen I, Craig WM, Boyce WF, et al. Associations between overweight and obesity with bullying behaviors in school-aged children. Pediatrics 2004;113:1187-1194.

8. Odar Stough C, Merianos A, Nabors L, et al. Prevalence and predictors of bullying behavior among overweight and obese youth in a nationally representative sample. Child Obes 2016;12:263-271.

9. Cook CR, Williams KR, Guerra NG, et al. Predictors of bullying and victimization in childhood and adolescence: A meta-analytic investigation. Sch Psychol Q 2010;25:65.

10. Álvarez-García D, García T, Núñez JC. Predictors of school bullying perpetration in adolescence: A systematic review. Aggress Behav 2015;23:126-136.

11. Jansen PW, Verlinden M, Dommisse-van Berkel A, et al. Teacher and peer reports of overweight and bullying among young primary school children. Pediatrics 2014;134:473-480.

12. Bacchini D, Licenziati MR, Garrasi A, et al. Bullying and victimization in overweight and obese outpatient children and adolescents: An Italian multicentric study. PLoS One 2015;10:e0142715.

13. Srabstein JC, McCarter RJ, Shao C, et al. Morbidities associated with bullying behaviors in adolescents. School based study of American adolescents. Int $J$ Adolesc Med Health 2006;18:587596.

14. Bogart LM, Elliott MN, Klein DJ, et al. Peer victimization in fifth grade and health in tenth grade. Pediatrics 2014;133:440-447.

15. Juvonen J, Graham S, Schuster MA. Bullying among young adolescents: The strong, the weak, and the troubled. Pediatrics 2003; 112:1231-1237.

16. U.S. Department of Health and Human Services HRaSAH, Maternal and Child Health Bureau (MCHB). 2016 National Survey of Children's Health (2016), Sampling and Survey Administration. Child and Adolescent Health Measurement Initiative (2016). 2016; Available at www.childhealthdata.org Last accessed August 14, 2018.

17. United States Census Bureau, Associate Director of Demographic Programs, National Survey of Children's Health. 2016 National Survey of Children's Health frequently asked questions. Available at https://mchb.hrsa.gov/data/national-surveys/data-user Last accessed August 14, 2018.

18. World Health Organization. Adolescent Health. Available at www.who.int/topics/adolescent_health/en Last accessed August 14, 2018.

19. Barlow SE, Expert C. Expert committee recommendations regarding the prevention, assessment, and treatment of child and 
adolescent overweight and obesity: Summary report. Pediatrics 2007;120 Suppl 4:S164-S192.

20. Lebrun-Harris LA, Sherman LJ, Limber SP, et al. Bullying victimization and perpetration among US children and adolescents: 2016 National Survey of Children's Health. J Child Fam Stud 2018:1-15.

21. Timmermanis V, Wiener J. Social correlates of bullying in adolescents with attention-deficit/hyperactivity disorder. Can J Sch Psychol 2011;26:301-318.

22. Carbone-Lopez K, Esbensen F-A, Brick BT. Correlates and consequences of peer victimization: Gender differences in direct and indirect forms of bullying. Youth Violence Juv Justice 2010; $8: 332-350$.

23. Nigg JT, Johnstone JM, Musser ED, et al. Attention-deficit/ hyperactivity disorder (ADHD) and being overweight/obesity: New data and meta-analysis. Clin Psychol Rev 2016;43: 67-79.

24. Taylor LA, Saylor C, Twyman K, et al. Adding insult to injury: Bullying experiences of youth with attention deficit hyperactivity disorder. Child Health Care 2010;39:59-72.

25. Russell-Mayhew S, McVey G, Bardick A, et al. Mental health, wellness, and childhood overweight/obesity. J Obes 2012;2012: 281801.

26. Sentenac M, Arnaud C, Gavin A, et al. Peer victimization among school-aged children with chronic conditions. Epidemiol Rev 2011; 34:120-128.

27. Strauss RS, Pollack HA. Social marginalization of overweight children. JAMA Pediatr 2003;157:746-752.

28. Van Geel M, Vedder P, Tanilon J. Are overweight and obese youths more often bullied by their peers? A meta-analysis on the relation between weight status and bullying. Int J Obes 2014;38: 1263.

29. Eisenberg ME, Neumark-Sztainer D, Story M. Associations of weight-based teasing and emotional well-being among adolescents. Arch Pediatr Adolesc Med 2003;157:733-738.

30. Akinbami LJ, Ogden CL. Childhood overweight prevalence in the United States: The impact of parent-reported height and weight. Obesity 2009;17:1574-1580.

31. U.S. Department of Health and Human Services CfDCaP, National Center for Health Statistics. Design and Operation of the National Survey of Children's Health, 2007. Vital and Health Statistics 2012; 1. Available at www.cdc.gov/nchs/data/series/sr_01/sr01_055.pdf Last accessed August 14, 2018

32. O'Connor DP, Gugenheim JJ. Comparison of measured and parents' reported height and weight in children and adolescents. Obesity 2011;19:1040-1046.

33. Copeland WE, Wolke D, Angold A, et al. Adult psychiatric outcomes of bullying and being bullied by peers in childhood and adolescence. JAMA Psychiatry 2013;70:419-426.

Address correspondence to:

Kristie Rupp, PhD

Department of Kinesiology

Brooklyn College of the City University of New York 2900 Bedford Avenue 428 West Quad Building Brooklyn, NY 11210

E-mail: kristie.rupp@brooklyn.cuny.edu 